\title{
The Nature of Galactic Bulges from SAURON Absorption Line Strength Maps
}

\author{
Reynier F. Peletier ${ }^{1}$, Jesús Falcón-Barroso ${ }^{2}$, Katia Ganda ${ }^{1}$, \\ Roland Bacon $^{3}$, Michele Cappellari ${ }^{4}$, Roger L. Davies ${ }^{4}$, \\ P. Tim de Zeeuw ${ }^{5}$, Eric Emsellem ${ }^{3}$, Davor Krajnović ${ }^{4}$, \\ Harald Kuntschner ${ }^{6}$, Richard M. McDermid ${ }^{5}$, Marc Sarzi ${ }^{7}$ \\ and Glenn van de Ven $^{8}$
}

\footnotetext{
${ }^{1}$ Kapteyn Astronomical Institute, University of Groningen, NL-9700 AV Groningen email: peletier@astro.rug.nl

${ }^{2}$ European Space Agency/ESTEC, Keplerlaan 1, NL-2200 AG Noordwijk,

${ }^{3}$ Observatoire de Lyon, 9 av. Charles André, F-69230 Saint-Genis Laval,

${ }^{4}$ Sub-Department of Astrophysics, University of Oxford, Oxford OX1 3RH, UK,

${ }^{5}$ Sterrewacht Leiden, University of Leiden, NL-2333 CA Leiden,

${ }^{6}$ ST-ECF, European Southern Observatory, D-85748 Garching bei München,

${ }^{7}$ Centre for Astrophysics Research, University of Hertfordshire, Hatfield, UK,

${ }^{8}$ Institute for Advanced Study, Einstein Drive, Princeton, NJ 08540, USA
}

\begin{abstract}
We discuss SAURON absorption line strength maps of a sample of 24 early-type spirals, mostly Sa. From the Lick indices $\mathrm{H} \beta, \mathrm{Mg} b$ and $\mathrm{Fe} 5015$ we derive SSP-ages and metallicities. By comparing the scaling relations of $\mathrm{Mg} b$ and $\mathrm{H} \beta$ and central velocity dispersion with the same relation for the edge-on sample of Falcón-Barroso et al. (2002) we derive a picture in which the central regions of Sa galaxies contain at least 2 components: one (or more) thin, disclike component, often containing recent star formation, and another, elliptical-like component, consisting of old stars and rotating more slowly, dominating the light above the plane. If one defines a bulge to be the component responsible for the light in excess of the outer exponential disc, then many Sa-bulges are dominated by a thin, disc-like component containing recent star formation.
\end{abstract}

Keywords. galaxies: spiral, galaxies: stellar content, galaxies: bulges

\section{Introduction}

The measurement of absorption line strengths in combination with stellar population models has been used for many years to probe the ages and metallicities from integrated stellar populations of galaxies. Although there exist many studies of elliptical galaxies and S0's, absorption line studies of spiral galaxies are lagging behind. They are, however, important to understand the origin of galactic bulges. Are bulges old, elliptical-like objects in the middle of a large, spiral disk, which formed first? Or are they mass and light concentrations that formed from internal processes in the disk? Recent reviews about this topic are given by Kormendy \& Kennicutt (2004) and Athanassoula (2005). Stellar population studies of early-type spirals (Jablonka et al. 1996, Proctor \& Sansom 2002) see very little difference between the central stellar populations of spirals and S0's. Both studies, however, contain few fainter galaxies with a central velocity dispersion smaller than $120 \mathrm{~km} / \mathrm{s}$. Those objects show stellar populations with a variety of properties (Moorthy \& Holtzman 2006). At present it looks as if both types of bulges (elliptical-like and disk-like) exist. It is not clear, however, why this is, and how the bulge-type is related to its stellar populations. 


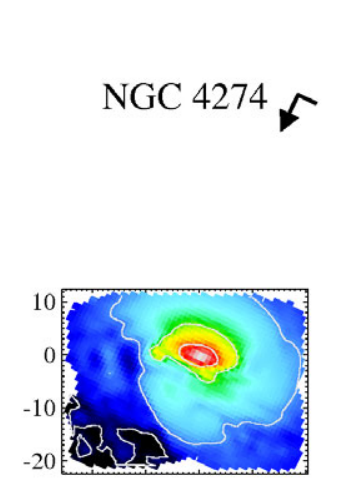

Cont. Flux

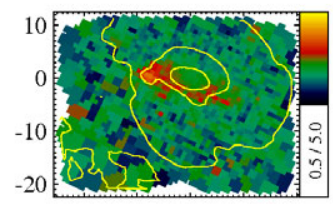

$\mathrm{H} \beta$

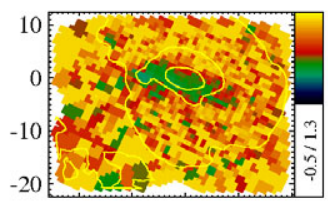

Age

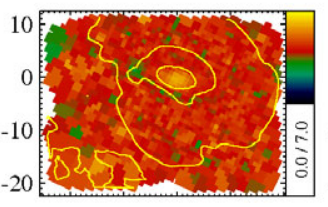

Fe 5015

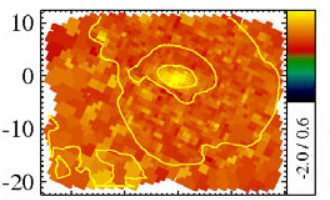

$\mathrm{Z}$

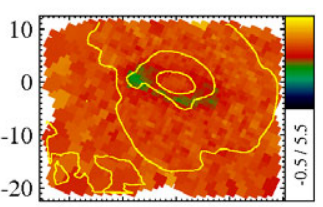

$\mathrm{Mg} \mathrm{b}$

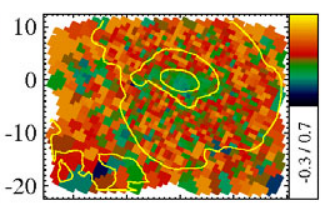

$[\mathrm{Mg} / \mathrm{Fe}]$

Figure 1. Absorption line strength maps of one of the galaxies. Shown are (from left to right): line indices $\mathrm{H} \beta$, Fe 5015 and $\mathrm{Mg} b$. Second row: Reconstructed intensity, logarithmic Age, Metallicity $\left(\log \mathrm{Z} / \mathrm{Z}_{\odot}\right)$ and $[\alpha / \mathrm{Fe}]$. The reconstructed intensity is overlaid in white contours on the maps.

In this work we present high quality, two-dimensional absorption line maps of a sample of 24 early-type spirals. Here some highlights of the work are given. More details are found in Peletier et al. (2007).

\section{SAURON absorption line strength maps of Sa galaxies}

We have obtained Integral Field Spectroscopy in a field of $33^{\prime \prime} \times 41^{\prime \prime}$, with a spatial sampling of $0.94^{\prime \prime} \times 0.94^{\prime \prime}$ using SAURON at the WHT in La Palma. The observations are part of the SAURON survey, described in de Zeeuw et al. (2002). The sample consists of 24 early-type spiral galaxies, for which the kinematics of gas and stars have been presented in Falcón-Barroso et al. (2006). The spectra, which have a wavelength range from 4790 to $5300 \AA$, were fitted with the stellar population models of Vazdekis (1999), allowing us to separate the emission lines from the absorption line spectrum (for details about this procedure see Sarzi et al. 2006 and Falcón-Barroso et al. 2006). From the cleaned spectra we obtained the line indices $\mathrm{H} \beta, \mathrm{Mg} b$ and $\mathrm{Fe} 5015$.

In the way we described in Kuntschner et al. (2006) we determined ages, metallicities and abundance ratios at every position, assuming that the stellar populations there could be represented by a single-age, single metallicity stellar population. In practise, we determined the SSP for which the line strengths $\mathrm{Fe} 5015, \mathrm{H} \beta$ and $\mathrm{Mg} b$ fitted best in the $\chi^{2}$ sense (Fig. 1). Although we know that it is a great over-simplification to represent the stellar populations (even locally) of a galaxy by a SSP, in some, especially elliptical galaxies this approach gives a good first-order approximation.

\section{Relations between indices and velocity dispersion}

Early-type galaxies show a tight $\mathrm{Mg}_{2}-\sigma$ relation (Terlevich et al. 1981). Deviations from the relations correlate well with parameters indicating the presence of young stellar populations. In Falcón-Barroso et al. (2002) we used the relation to show that the stellar populations in a sample of inclined early-type spirals are generally old. In Fig. 2 we show the central $\mathrm{Mg} b$ and $\mathrm{H} \beta$ indices of our sample as a function of the central velocity dispersion $\sigma_{\text {cen }}$. In the figure are shown the galaxies of this sample, together with the 

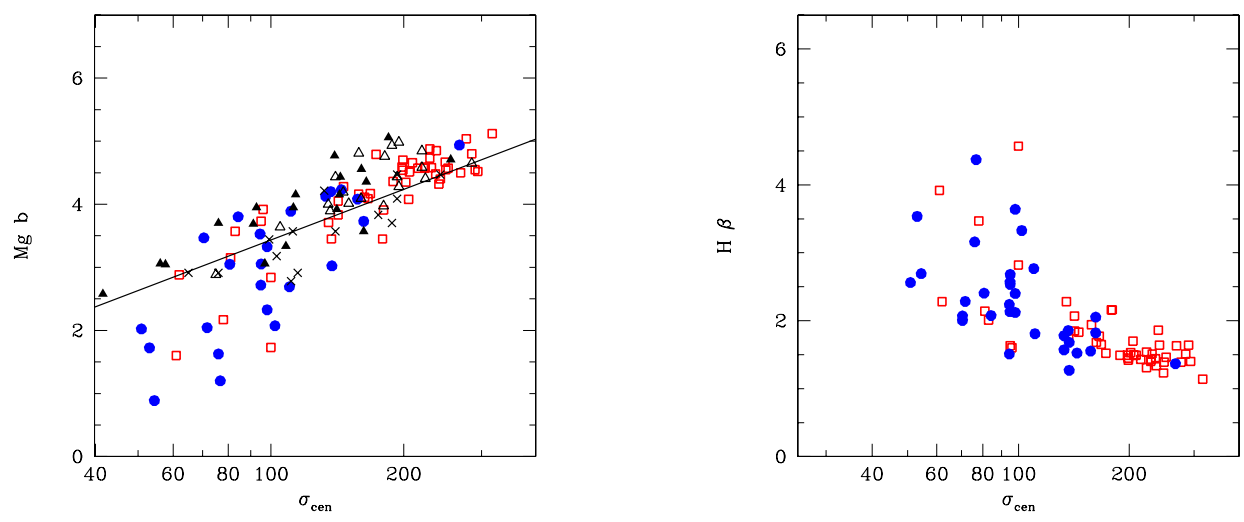

Figure 2. Left: Central indices as a function of central velocity dispersion (in $\mathrm{km} / \mathrm{s}$ ). The open red symbols show the ellipticals and S0 galaxies of Kuntschner et al. (2006) for an aperture of $r_{e} / 8$. The black line is the least-squares fit to the ellipticals and S0 galaxies in Coma of Jørgensen et al. (1996). As a comparison we also show a few literature samples in black: the highly-inclined bulges of FB02 (filled triangles), the bulges of Bender et al. (1993, open triangles), and bulges of Jablonka et al. (1996, crosses).

ellipticals and lenticulars of Kuntschner et al. (2006) (at $\mathrm{r}_{e} / 8$ ), and a number of literature samples of early-type spirals (see caption). The black line is a best fit to the ellipticals and S0 galaxies in the Coma cluster of Jørgensen et al. (1996). The $\mathrm{Mg} b-\sigma$ relation of elliptical galaxies and S0's acts as an upper envelope for the Sa galaxies. Although some Sa galaxy centre measurements lie close to the relation, a significant fraction of the galaxies falls below it. The same effect is seen for the $\mathrm{H} \beta-\sigma$ relation. Using the argumentation of Schweizer et al., the line of galaxies in Coma would correspond to old stellar populations, while deviations would be caused by younger stars. The fact that our Sa bulges mostly lie below the $\mathrm{Mg} b-\sigma$ relation or above the $\mathrm{H} \beta-\sigma$ relation would indicate that the centres of Sa bulges generally are significantly younger than early-type galaxies in the Coma cluster. This result appears to contradict the tight $\mathrm{Mg}_{2}-\sigma$ relation for bulges found by FB02 and also the relation by Jablonka et al. (1996). It confirms, however, the results of Prugniel et al. (2001), who find several early-type spiral galaxies lying considerably below the $\mathrm{Mg}_{2}-\sigma$ relation. Notice that there are several $\mathrm{S} 0$ galaxies that are far away from the relation defined by elliptical galaxies, in the same location as the spirals with the lowest $\mathrm{Mg} b$ values.

\section{Star formation histories in the central regions of early-type spirals}

In the region of interest $(\sigma<120 \mathrm{~km} / \mathrm{s})$ the galaxies of FB02 generally have higher $\mathrm{Mg} b$ than the galaxies of this sample. Why this difference? The only important difference between the two samples is the inclination distribution. If Sa galaxies would contain young stellar populations that would be situated in the plane, we would see them in the SAURON sample. In FB02, however, where we looked at $5^{\prime \prime}$ from the center on the minor axis, we would not have seen them, if the young stellar populations were limited to the very central regions. Since for these Sa galaxies the light in the central regions is completely dominated by the bulge, it seems that bulges consist of 2 , maybe more components: one which is old, elliptical-like, and slowly rotating, and another more flattened, disk-like component containing often young stellar populations. Support to this idea is given by the fact that the SAURON maps show that the young stellar populations 
can often be found in circumnuclear rings, features with a small vertical extent. Another supporting argument is the fact that many of the galaxies have local central velocity dispersion minima (Falcón-Barroso et al. 2006, see also Ganda et al. 2006, Emsellem et al. 2001). These are most likely caused by central discs, some of which contain young stellar populations.

According to the current literature there are several kinds of bulges. Bulges that photometrically $\left(\mathrm{r}^{1 / 4}\right.$ surface brightness law) and kinematically (still relatively high $\lambda_{R}$, Emsellem et al. 2007) resemble elliptical galaxies are often called classical bulges (Kormendy \& Kennicutt 2004). A bulge consisting only of the fast-rotating component is called a pseudo-bulge in this reference. Athanassoula (2005) claims that there are three types of bulges: the classical bulges, which form by collapse or merging, disc-like bulges, which result from the inflow of (mainly) gas to the centre-most parts, and subsequent star formation, and boxy and peanut bulges, which are seen in near-to-edge-on galaxies and which are in fact just a part of the bar seen edge-on, and therefore not part of the bulge in the definition of this paper. Here we add another piece of the puzzle. From the stellar population distribution, by comparing a sample uniformly distributed in inclination with a sample biased towards high inclination we infer that galactic bulges have more than one physical component: generally they have a slowly-rotating, elliptical-like component, and one or more fast-rotating components in the plane of the galaxy. This picture also nicely explains the fact that bulge populations in general are very similar to those in the disc (e.g. Peletier \& Balcells 1996).

\section{References}

Athanassoula, E. 2005, MNRAS, 358, 1477

Bender, R., Burstein, D., \& Faber, S. M., 1993, ApJ, 411, 153

de Zeeuw, P. T., et al., 2002, MNRAS, 329, 513

Emsellem, E., Greusard, D., et al., 2001, A\&A, 368, 52

Emsellem, E., Cappellari, M. Krajnovic, D., et al., 2007, MNRAS, submitted

Falcón-Barroso, J., Peletier, R. F., \& Balcells, M., 2002, MNRAS, 335, 741, FB02

Falcón-Barroso, J., et al. 2006, MNRAS, 369, 529

Ganda, K., Falcón-Barroso, J., Peletier, R. F., et al., 2006, MNRAS, 367, 46

Jablonka, P., Martin, P., \& Arimoto, N., 1996, AJ, 112, 1415

Jørgensen, I., Franx, M., \& Kjaergaard, P., 1996, MNRAS, 280, 167

Kormendy, J., \& Kennicutt, R. C., 2004, ARA\&A, 42, 603

Kuntschner, H., et al., 2006, MNRAS, 369, 497

Moorthy, B. K., \& Holtzman, J. A. 2006, MNRAS, 371, 583

Peletier, R. F., \& Balcells, M. 1996, AJ, 111, 2238

Peletier, R. F., et al., 2007, MNRAS, sbmitted.

Proctor, R. N., \& Sansom, A. E., 2002, MNRAS, 333, 517

Prugniel, P., Maubon, G., \& Simien, F., 2001, A\&A, 366, 68

Sarzi, M., et al. 2006, MNRAS, 366, 1151

Schweizer, F., Seitzer, P., et al., 1990, ApJ, 364, L33

Terlevich, R., Davies, R. L., Faber, S. M., \& Burstein, D. 1981, MNRAS, 196, 381

Trager, S. C., Faber, S. M., Worthey, G., \& González, J. J., 2000, AJ, 119, 1645

Vazdekis, A. 1999, ApJ, 513, 224 